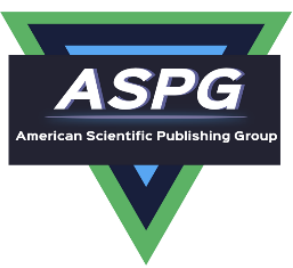

\title{
Characterizing wavelet coefficients with decomposition for medical images
}

\author{
Jabbar Abed Eleiwy \\ ${ }^{1}$ Applied Science Department, University of Technology, Baghdad, Iraq, e-mail: \\ 100310@uotechnology.edu.iq,
}

\begin{abstract}
In this paper, applications Discrete Laguerre Wavelet Transform were used where satisfactory results were obtained, where the efficiency of our proposed theory was proved and the examples used will prove this. Three physical samples were selected that were compressed using the proposed wavelets, and good results were obtained that prove the efficiency of the method used.

three physical samples were selected that were compressed using the proposed wavelets, and good results were obtained that prove the efficiency of the method used.
\end{abstract}

Keywords: Discrete Laguerre Wavelet Transform (DLWT), Image Compression, Medical application, De-noise medical image, bit per pixel

\section{Introduction}

The compression of the color image as described in many works means data compression or bit rate reduction on cryptographic information using bits lower than the original representation. This is an important technique in the field of image processing and transmission of information where the bit rate reduction rate is based on original image information or encryption information In order to reduce the storage space so that the important benefits of pressure is to minimize the potential loss of data where the identification and elimination of statistical repetition This technique in information theory is the number of bits used to send a message minus the number of bits of information Effective in the message [1-6].

Where many algorithms are used to explain how to work with this technique to perform data compression without loss and to obtain good results when rebuilding and return to the original data without loss, the error rate is almost equal to zero through the application mean square error and Peak signal-to-noise ratio [7-11] The following technique DLWT [9-11], was used to implement the technique mentioned above and apply it to a color image in which the account Bit-per-pixel was reached. In the following sections, the proposed theory was based on a section of a mammalian mammogram that was examined with magnetic resonance imaging.

DOI: $10.5281 /$ zenodo. 5182849

Received: March 09, 2021 Accepted: July 20, 2021 
The reset of this paper is organized as follows: Section 2 describes the proposed transform model. Section 3 explains the data compression process. Also, sections three discusses the results and the analysis. Section 4 concludes the paper.

\section{Proposed Transform model}

The following family function

$$
\begin{aligned}
& \rho_{s, r}(t)=|s|^{\frac{-1}{2}} \rho\left(\frac{t-r}{s}\right) \\
& s, r \in R, \quad s \neq 0
\end{aligned}
$$

where

$$
\rho(t)=\left[\rho_{0}(t), \rho_{1}(t), \ldots, \rho_{M-1}\right]^{T}
$$

The elements $\rho_{0}(t), \rho_{1}(t), \ldots, \rho_{M-1}(t)$ are the basis functions, orthogonal on the $[0,1]$.

\section{Data Compression: [12-14]}

The primary property is the relative characteristics of the relative scarcity of the wavelet representation of the signal

The following items can be used accurately

1. Rounding coefficients for which a small number can be used

2- detail processing such as noise removal

We can perform the compression process in three steps

1 - The selection of the wavelet is analyzed

2- The level at which the work is done is $\mathrm{N}$

3- The signal is analyzed at a certain level and after setting the threshold value at the lower level N-1

Starting from level $\mathrm{N}$, the reconstruction of the wavelet is done using the original approximate coefficients

Tow compressions approaches available

1- In this case, the wavelet expansion of the signal is taken while maintaining the largest absolute value coefficients where a global threshold is possible

2- The application is visually level where it depends on the maximum. For each 1-n level, detail coefficients are used

\subsection{Image Compression [[15-16]}

in this section compression image for example the noise woman $(96 \times 96)$ selected the level threshold in level 1 (20.64) and in level 2 (32.3) with returned energy 99.59\% and number of zero $53.98 \%$ if we compared with global threshold the above results are better than there the following tow figures show it

DOI: $10.5281 /$ zenodo. 5182849

Received: March 09, 2021 Accepted: July 20, 2021 


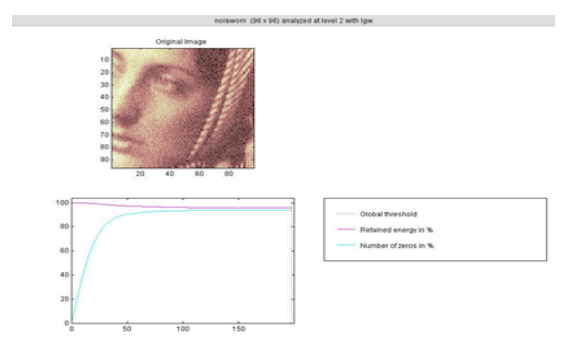

Figure.1 compressing image with global thresholding using DWT

The above figure shows compressing image with global thresholding by using DLWT with returned energy $95.84 \%$, the number of zero is 93.75 . The following figure shows compressing image with threshold by level.
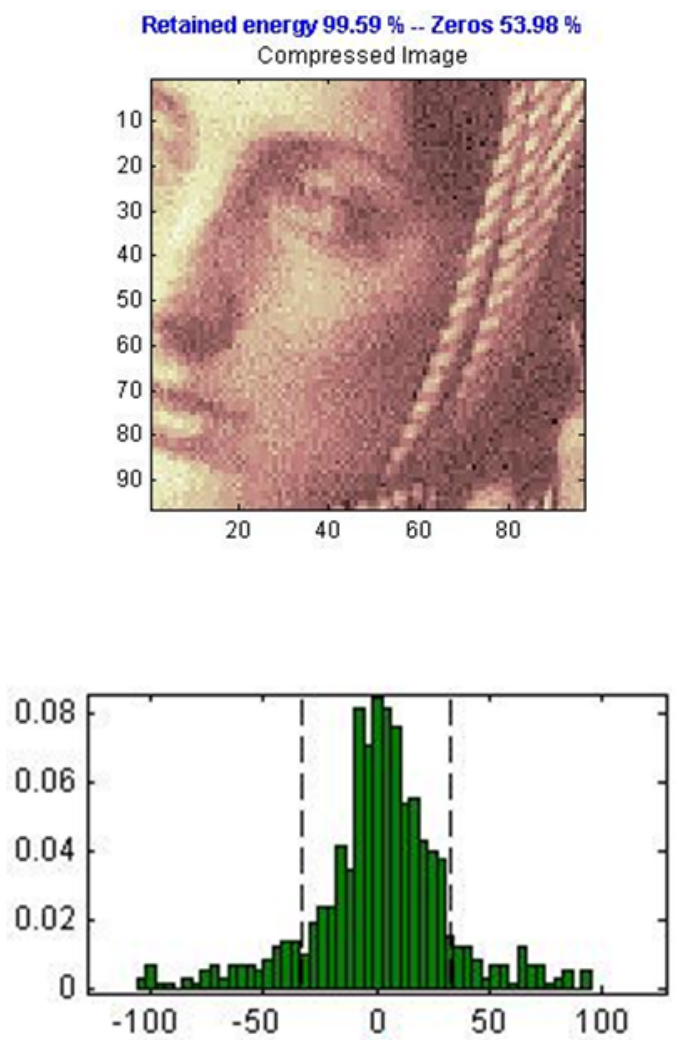

Figure. 2 compressing image with thresholding by level by using DWT 


\subsection{Two-Dimensional True Compression}

Wavelet Toolbox software (WTBS) features two-dimensional true compression features and more information on compression methods. We start this process from the image you want to work on. The purpose of the compression is to reduce the length of the bit string needed to represent it, Information where the task of wavelets is to find effective solutions to this problem.

In this section, the real color image was treated. The image was compressed using DLWT and the quantization stages were followed. The compression and decomposition of the color image was illustrated using different methods. The global threshold was used with the Hoffmann markup.

The following algorithm shows how performance the above processed

Algorithm.1: Two-Dimensional True Compression

Input : image $(\mathrm{X})$

Output : compressed the image by using DLWT

Step 1: load image (Mask)

Step 2: In this step the process of giving synthetic pressure is done by pressure and bit rate per pixel

Step 3: The basic colors are (RGB). By using the new transformations of the wavelets, the process is done in the plane (2) then.

$$
\begin{aligned}
& \mathrm{CR}=6.69 \\
& \mathrm{BPP}=0.54
\end{aligned}
$$

\subsection{Medical application}

In the eighth chapter, the process of compressing the medical images taken under the magnetic resonance of breast cancer was clarified. In this section, other bone images will be shown to show bone necrosis
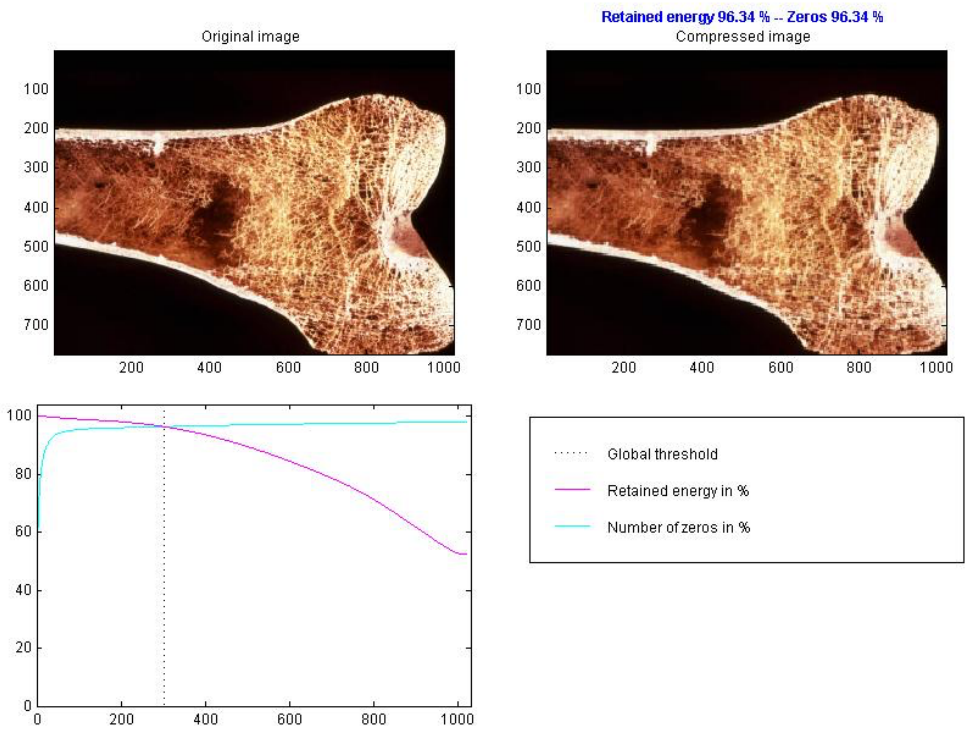

Figure. 3 compressed boon's image with global threshold 302.5 retained energy $96.34 \%$ - zeros $96.34 \%$ 

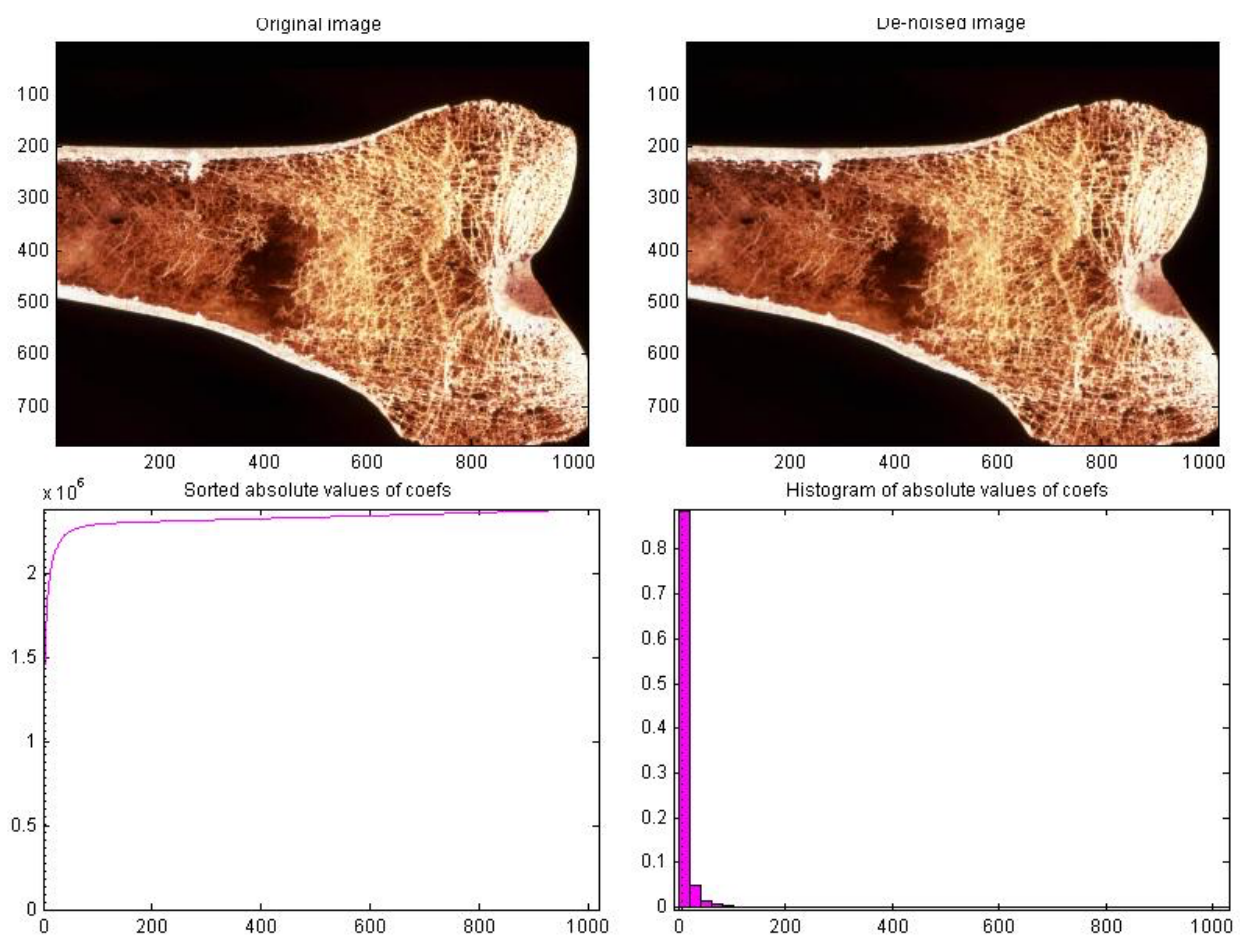

Figure.4 De-Noising boon's image with soft threshold 5.365
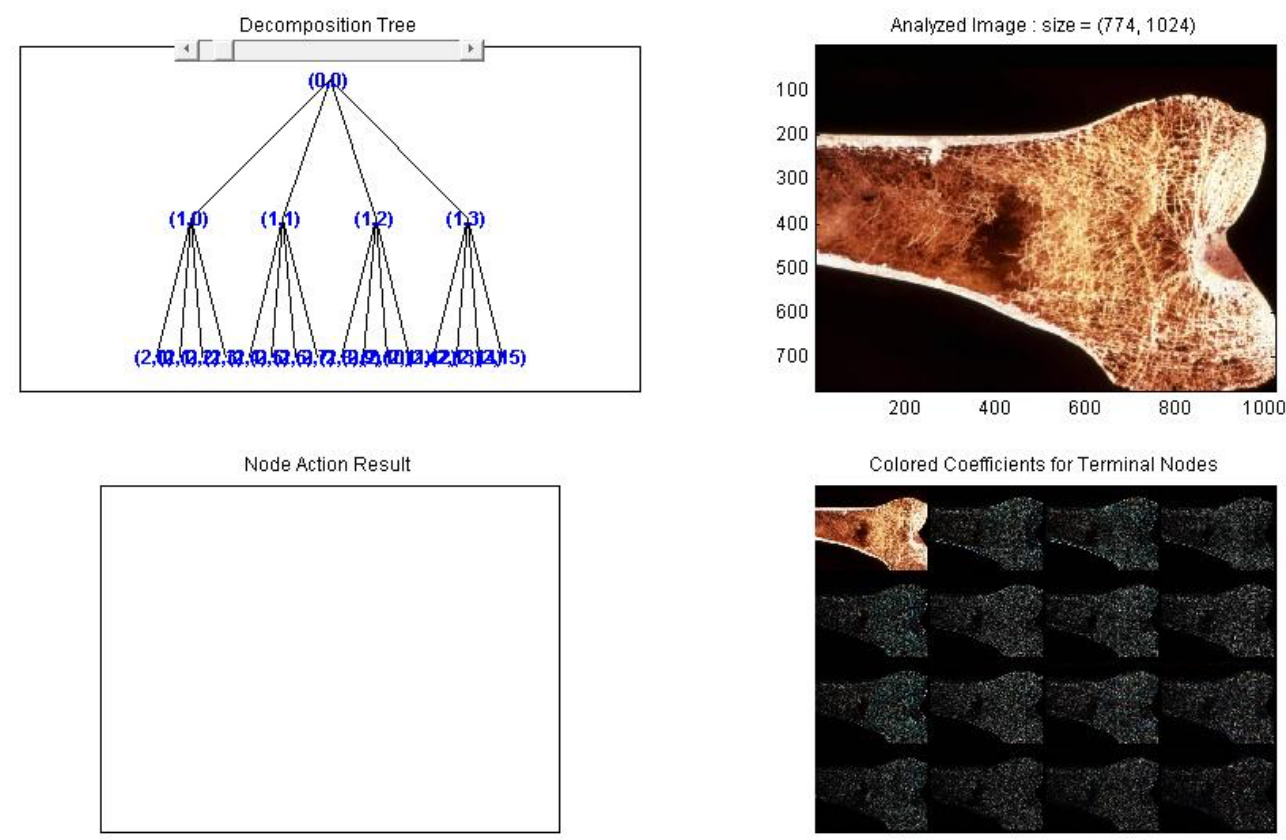

Colored Coefficients for Terminal Nodes

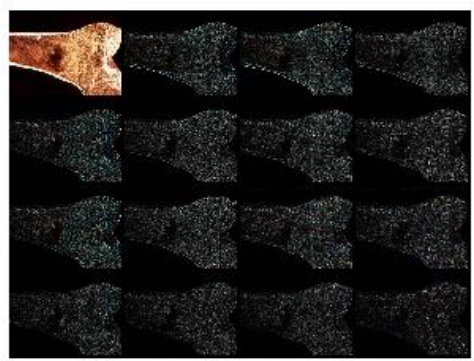

Figure. 5 analyses boon's image with initial tree 


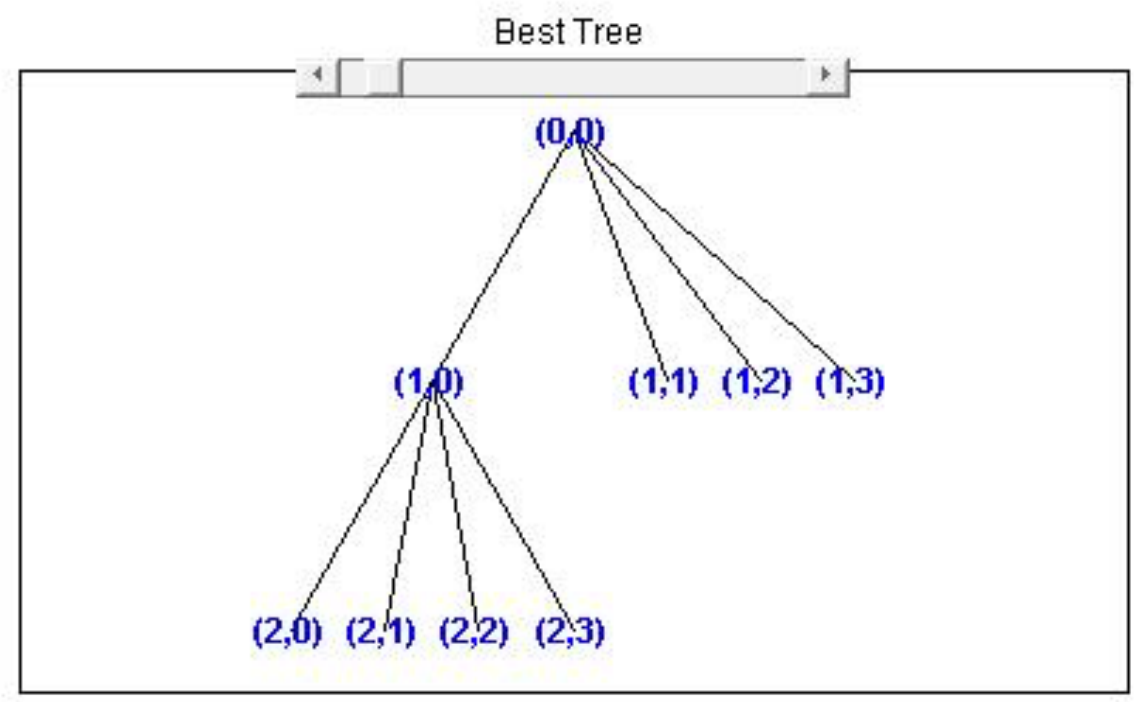

Figure. 6 analyses boon's image with Best tree

Colored Coefficients for Terminal Nodes

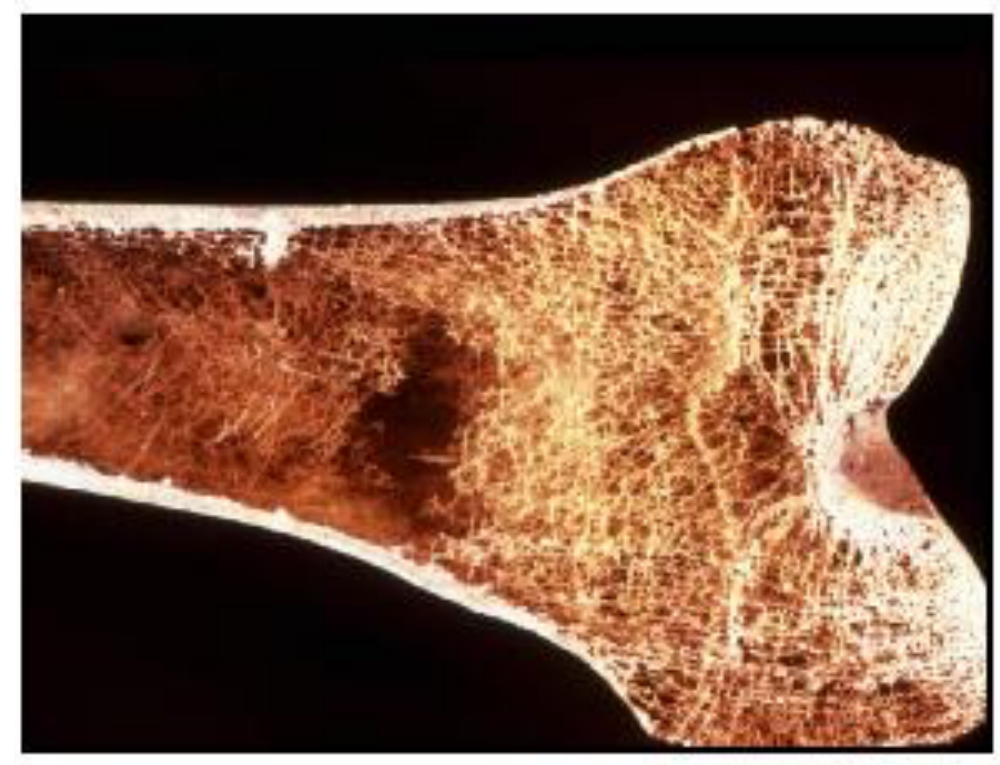

Figure.7 analyses boon's image with Best level 


\section{Conclusions}

In this work, the normal color image and the medical images that were examined with Magnetic resonance imaging (MRI) were compressed using DLWT and the results indicated in the tables above proved the efficiency of the theory used, allowing it to be used in many fields such as medicine, Engineering and Sciences.

\section{References}

1. Andreas Savakis and Richard Carbone, 'Discrete Wavelet Transform Core for Image Processing Application' SPIE, Vol 5671, 142-151, (2005) .

2. B.R. Ambedkar \& Sangeeta Arora 'Numerical Solution of Wave Equation Using Haar Wavelet', International Journal of Pure and Applied Mathematics (IJPAM), VOL (98), NO (4), PP 457-469, (2015).

3. B. Satyanarayan, Y. Pragathi Kumar, Asma Abdulelah, 'Laguerre Wavelet and its Programming' , International Journal of Mathematics Trends and Technology (IJMTT), VOL(49), NO(2), 129-137), (2017).

4. B. Satyanarayan, Asma Abdulelah 'Mathematical Aspects of Laguerre Wavelets Transformation', Annals of Pure and Applied Mathematics, (APAM), VOL(16), NO(1), PP(53-61), (2018).

5. Debayan Goswami, 'A Discrete Wavelet Transform based Cryptographic algorithm', International Journal of Computer Science and Network Security (IJCSNS), Vol,11,No4,(2011)

6. Dipalee Gupta, Siddhartha Choubey 'Discrete Wavelet Transform for Image Processing', International Journal of Emerging Technology and Advanced Engineering, (IJETAE), VOL(4), PP(598-602), (2015).

7. Juanli HU, Jiabin Deng, 'Image Compression Based on Improved FFT Algorithm'Journal of Networks, VOL(6), NO(7), PP(1041-1048), (2011).

8. Kamrul Hasan, Koichi Harada, 'Haar Wavelet Based Approach for Image Compression and Quality Assessment of Compressed Image', International Journal of Applied Mathematics (IJAM), VOL(36), NO(1), PP(1-8), (2007).

9. Lenka Kormanikora, 'Shape Design and Analysis of Adaptive Structures', Structural and Physical Aspects of Construction Engineering (ELSEVIER), VOL(190), PP(7-14), (2017).

10. Michael Berry, 'An Introduction to Wavelet Analysis', AMERICAN MATHEMATICAL SOCIETY, VOL(40), NO(3), PP(421-427), (2003).

11. Mridul Kumar, Gunjan Mathur, 'Image Compression Using DFT Through Fast Fourier Transform Technique', International Journal of Emerging Trends And Technology In Computer Science (IJETTCS), VOL(1), PP(129-133), (2012).

12. Michel Misiti, Y.Ves Misiti, 'Wavelets and Their Applications', Published by Hermes Science/ LAVOISER, (2003).

13. Muhammad Asad Iqbal \& Umer Saeed, 'Modified Laguerre Wavelets Method for Delay Differentail Equations of Fractional- Order', (Science Direct), VOL(2), PP(50-54), (2015).

14. Prabhjotot kour, 'Image Processing Using Discrete Wavelet Transform', International Journal of Electronics and Communication (IIJEC), VOL(3), NO(1), (2015).

15. Sanjeev Kumar, Varun Sood, 'Quality Assessment of Colour Image Compression Using Haar Wavelet Transform', International Journal of Engineering Trends and Technology (IJETT), VOL(3), NO(3), (2012).

16. Trevor.C. Bailey, 'Signal Detection in Underwater Sound Using Wavelets', Journal of the Amerirican Statistican Statistical Association, Vol. 93, No. 441. 73-83. (1998).

DOI: $10.5281 /$ zenodo. 5182849

Received: March 09, 2021 Accepted: July 20, 2021 